\title{
Effect of processing on morphology of hydroxyapatites: bioactive glasses and crystalline composites
}

Joel McAdams, Eric Bowman, Brian Cullum, Bradley Arnold, Lisa Kelly, et al.

Joel McAdams, Eric Bowman, Brian Cullum, Bradley Arnold, Lisa Kelly, Fow Sen Choa, N. B. Singh, Ching Hua Su, K. D. Mandal, Shruti Singh, "Effect of processing on morphology of hydroxyapatites: bioactive glasses and crystalline composites," Proc. SPIE 11020, Smart Biomedical and Physiological Sensor Technology XV, 1102006 (2 May 2019); doi: 10.1117/12.2516042

Event: SPIE Defense + Commercial Sensing, 2019, Baltimore, Maryland, United States 


\title{
Effect of processing on morphology of hydroxyapatites: Bioactive glasses and crystalline composites
}

\author{
Joel McAdams, Eric Bowman, Brian Cullum, Bradley Arnold, Lisa Kelly, Fow Sen Choa, and \\ N. B. Singh \\ University of Maryland, Baltimore County, 100 Hilltop Circle, Baltimore 21250 \\ Ching Hua Su \\ EM31, NASA Marshall Space Flight Center, Huntsville, AL 35812 \\ K.D. Mandal and Shruti Singh \\ Indian Institute of Technology, Banaras Hindu University, Varanasi (UP) India
}

Keywords: Composite, glass, bone, Annealing, Radiation, Crystallization, Coarsening

Email: singna@umbc.edu

\begin{abstract}
Recent studies on multinary oxides for applications as laser hosts and high dielectric capacitors have shown that processing at high temperature provides glassy or crystalline materials based on thermal treatments and cooling rates. Since hydroxyapatites are now subject of great interests due to their bioactivity, interest in producing soft and hard materials with glassy and crystalline nature by processing parameters has become very important. Crystalline materials by using Bridgman, Czochralski and flux growth methods are costly and require huge investment. We have observed that even low temperature solidification in organic flux produced oriented fibers. This organic treated material has different characteristics than in situ oxide materials prepared by sintering and grain growth. Examples of phosphate and silicate-based systems will be presented to demonstrate soft and hard materials. Effect of $\mathrm{TiO}_{2}$ and other hardening elements will be also reported.
\end{abstract}

\section{INTRODUCTION}

Bioactive glasses show considerable promise as bone tissue engineering scaffolds, which are utilized in processes such as bone regeneration and soft tissue repair [1-3]. These scaffolds must meet certain requirements in order to properly function, which include having high mechanical strength, porosity, and biocompatibility, making bioactive glasses ideal for this purpose. ${ }^{1}$ Bioactive glass particles and nanofibers have been shown to be able to promote wound healing, which is due in part to their high bioactivity, allowing them to tightly bind to bone and soft tissues. ${ }^{2,3}$ Bioactive glasses are also of particular interest due to their osteoconductivity [1]. Additionally, the dissolution of bioactive glasses results in angiogenesis, which is necessary for soft tissue repairs [2]. By controlling how bioactive glasses are prepared, characteristics that are desirable for bone tissue engineering can be better attained. The composition of bioactive glasses significantly impacts these characteristics. ${ }^{4}$ Some of the more well-known bioactive glasses include 4S5S, 13-93B3, and 1605, have different properties as a result of their compositions [2]. Bioactive glasses containing considerable amounts of boron, such as 13-93B3 and 1605, show potential as scaffolds, as boron promotes wound healing and helps prevent abnormal bone growth $[2,3]$. The properties of bioactive glasses are not only influenced by their composition but also by how they are synthesized [4]. Using higher temperatures can alter the structures of the resulting bioactive glasses. In this paper new bioactive glasses similar in composition to 1605 were synthesized and characterized to examine their properties as bioactive glasses for processes such as tissue scaffolding. These bioactive glasses included one comparable in composition to 1605 but containing manganese instead of magnesium (designated UMBG1, which stands for University of Maryland Bioglass \#1), another with a composition nearly identical to 1605 (designated UMBG2), and yet another that contained a significant amount of silicon dioxide (designated UMBG3). These bioactive glasses were synthesized as powders and were formed into pellets to test the durability of the material. Characterization techniques that were

Smart Biomedical and Physiological Sensor Technology XV, edited by Brian M. Cullum,

Douglas Kiehl, Eric S. McLamore, Proc. of SPIE Vol. 11020, 1102006 · (c) 2019

SPIE · CCC code: 0277-786X/19/\$18 - doi: 10.1117/12.2516042 
used included infrared (IR) and Raman spectroscopy, irradiation, differential thermal analysis, and scanning electron microscopy (SEM).

\section{EXPERIMENTAL METHODS}

2.1 Synthesis of Bioactive Glass Powders and Pellets: The starting materials were mixed together in stoichiometric amounts using a mortar and pestle. Due to the materials that were available, nearly all of the starting materials that are usually used to synthesize 1605 bioactive glass were replaced with different albeit similar compounds: $\mathrm{Na}_{3} \mathrm{PO}_{4}$ for $\mathrm{Na}_{2} \mathrm{O}$ and $\mathrm{P}_{2} \mathrm{O}_{5}, \mathrm{KOH}$ for $\mathrm{K}_{2} \mathrm{O}, \mathrm{CaCO}_{3}$ for $\mathrm{CaO}, \mathrm{B}(\mathrm{OH})_{3}$ for $\mathrm{B}_{2} \mathrm{O}_{3}$, and $\mathrm{MnO}_{4}$ or $\mathrm{MgSO}_{4}$ for $\mathrm{MgO}^{2}$ The amounts of these materials were adjusted accordingly for preparation of the bioactive glass powders. Copper oxide and zinc selenide were added as dopants to the mixture. The compositions of the bioactive glass powders created are listed in Table 1. After thoroughly mixing the starting materials together, the powder of each bioactive glass was compressed into a cylindrical pellet through the use of a mechanical pressing device, which can be seen in Figure 1. The pellets along with the powders of the bioactive glasses were then annealed in a $150{ }^{\circ} \mathrm{C}$ oven overnight and subsequently imaged through the use of a microscope. The pellets, which can be seen in Figure 2, were then annealed to $200{ }^{\circ} \mathrm{C}$ periodically, usually for a couple of days at a time, and were imaged with the microscope following each annealing. Note that the temperature of the $200{ }^{\circ} \mathrm{C}$ oven varied substantially depending on where the sample was placed in the oven, so these temperatures may not be exact.

Table 1. Composition by \% Weight of UMBG1, UMBG2, and UMBG3 Bioactive Glasses.

\begin{tabular}{|c|c|c|c|c|c|c|c|c|c|}
\hline \multicolumn{1}{|c|}{ Composition (\% wt) } \\
\hline $\begin{array}{c}\text { Bioactive } \\
\text { Glass }\end{array}$ & $\mathrm{Na}_{3} \mathrm{PO}_{4}$ & $\mathrm{KOH}$ & $\mathrm{Mn}_{3} \mathrm{O}_{4}$ & $\mathrm{MgSO}_{4}$ & $\mathrm{SiO}_{2}$ & $\mathrm{CaCO}_{3}$ & $\mathrm{~B}(\mathrm{OH})_{3}$ & $\mathrm{CuO}$ & $\mathrm{ZnSe}$ \\
\hline UMBG1 & 5.5 & 14.3 & 4.6 & - & - & 12.1 & 62.6 & 0.2 & 0.6 \\
\hline UMBG2 & 6.3 & 7.9 & - & 8.9 & - & 21.2 & 54.5 & 0.2 & 1.1 \\
\hline UMBG3 & 4.1 & 5.2 & - & 5.8 & 35.1 & 13.8 & 35.3 & 0.2 & 0.7 \\
\hline
\end{tabular}
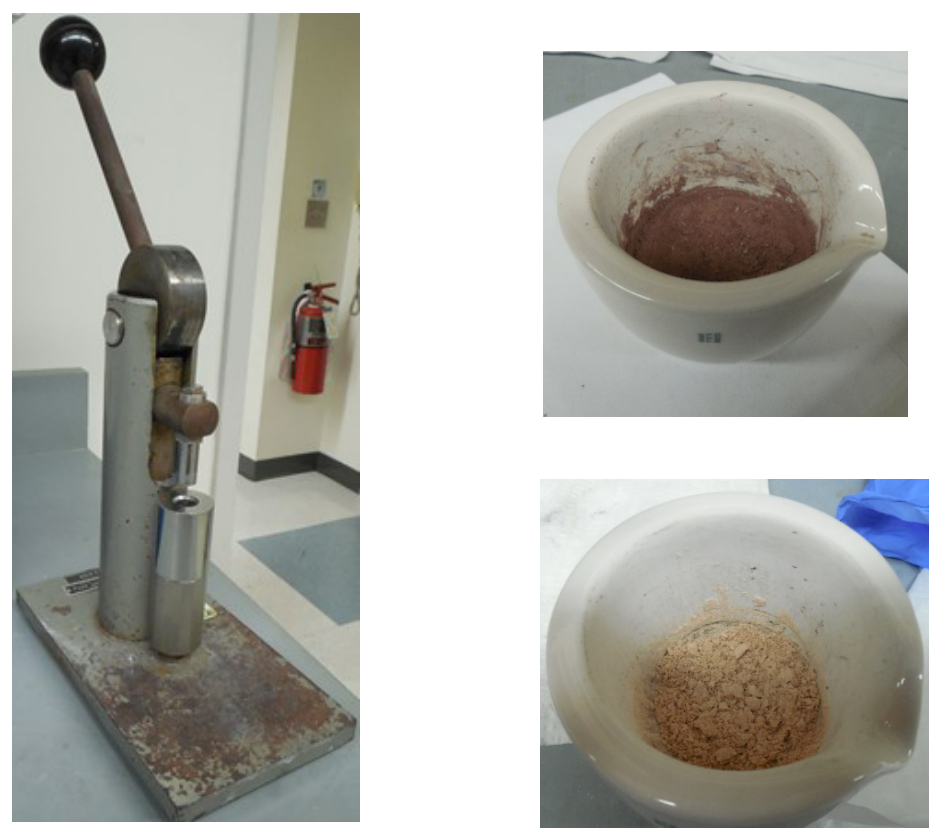

Figure 1. Left: Pressing machine used to form the pellets out of the bioactive glass powders. Right: Mortar used for mixing with powder of (top right) UMBG1 and (bottom right) UMBG2 bioactive glass powders. 

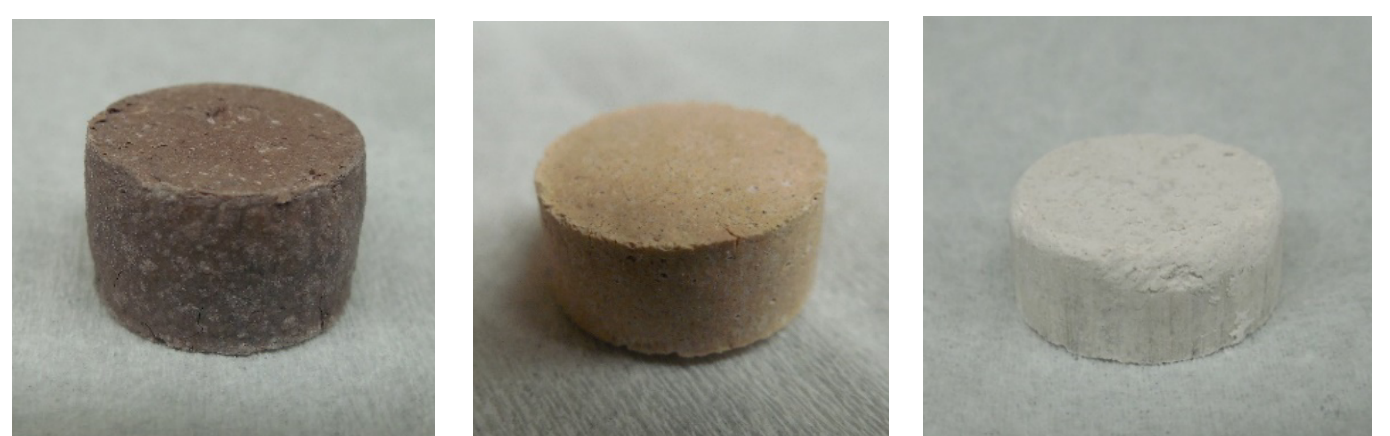

Figure 2. Bioactive glass $\mathrm{cm}$ size pellets after annealing at $200^{\circ} \mathrm{C}$. From left to right: UMBG1, UMBG2, and UMBG3.

2.2 Spectroscopic Characterization of Bioactive Glasses: A Mattson Genesis II FTIR instrument was used to collect IR spectra of the powders. A small amount of powder was placed on a potassium bromide salt plate, followed by the addition of a drop or two of dichloromethane (DCM) to help disperse the powder better on the plate. Another salt plate was then placed on top of the other one, which was subsequently set up on a stand. The IR spectra was then recorded from 400 to $4000 \mathrm{~cm}^{-1}$ with a resolution of $1 \mathrm{~cm}^{-1}$.

A Coherent brand Innova 70 argon ion laser and Acton SpectraPro 500i spectrometer cooled to $-120.0{ }^{\circ} \mathrm{C}$ using liquid $\mathrm{N}_{2}$ was used to obtain Raman spectra for the powders. The laser had to be set up, with silver mirrors and $514.5 \mathrm{~nm}$ notch filters being correctly positioned and the laser being properly aligned. The bioactive glass pellets were propped on an elevated platform and pressed closely against the microscope objective, allowing for backscattered Raman signals to be collected. The general set-up for the Raman experiments is displayed in Figure 3 and photographs of part of the set-up near the spectrometer can be seen in Figures 4. Raman spectra of the pellets were then compared to the compounds composing them, which was done by making annealed pellets out of the individual compounds and obtaining their spectra.

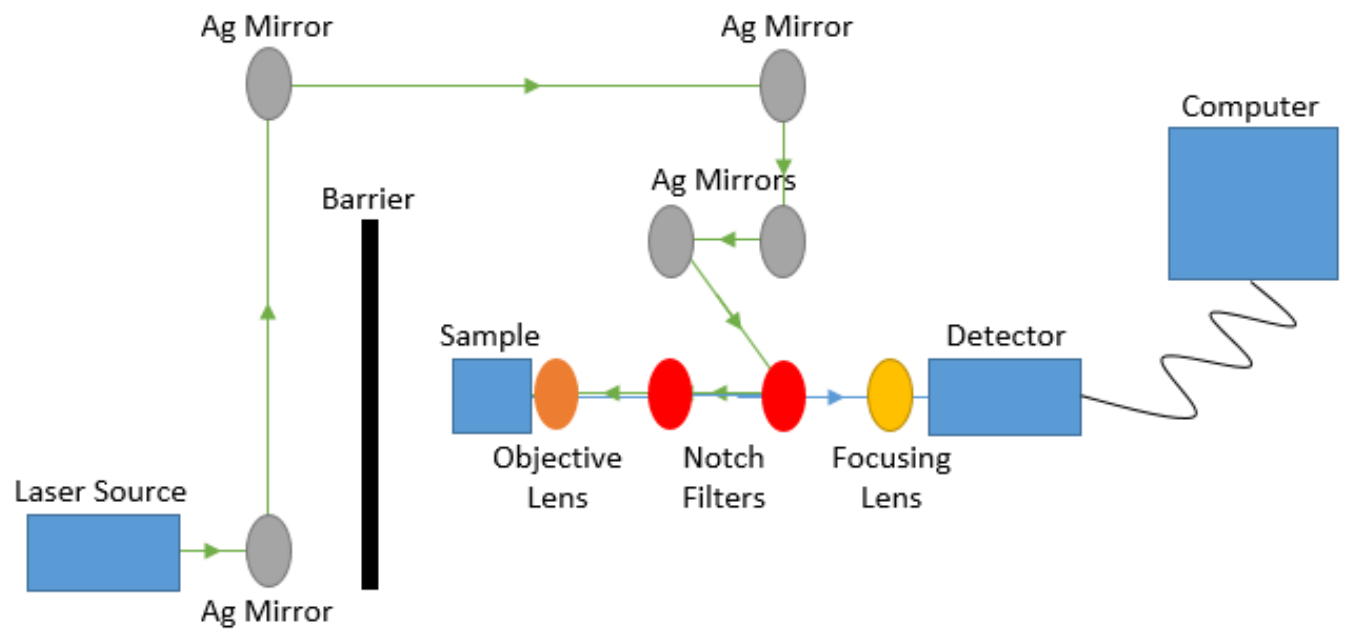

Figure 3. A simplified diagram of the laser set-up used for collecting Raman spectra. The "barrier" was equipment that was in the way, which meant additional silver mirrors were required to redirect the laser beam. 


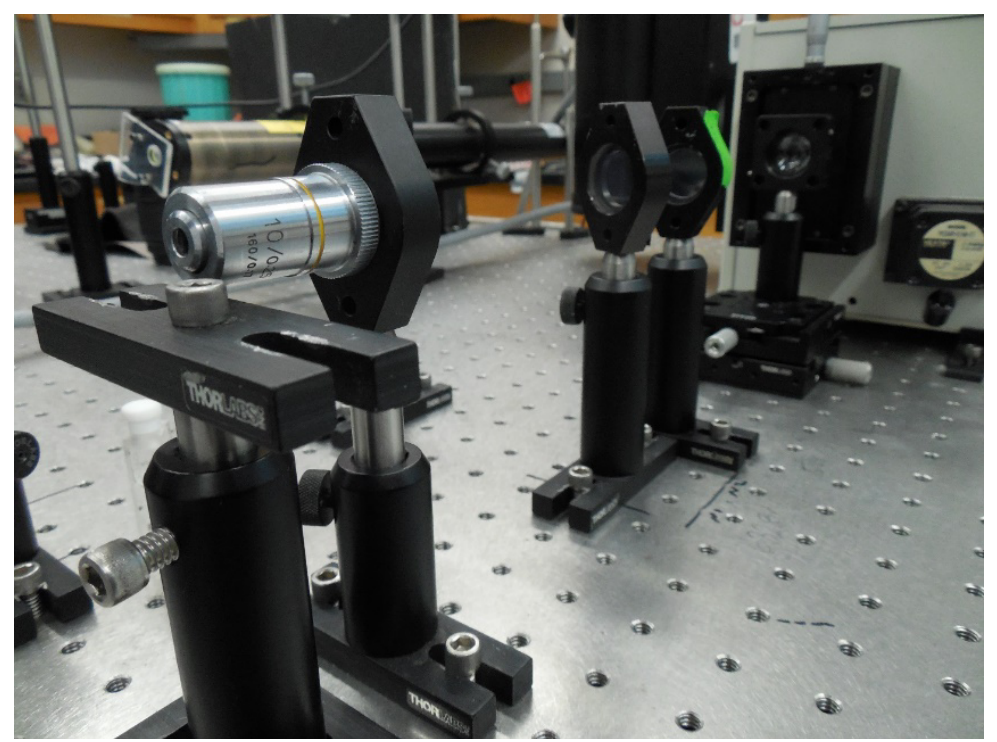

Figure 4. Close-up view of the Raman spectroscopy set-up near the spectrometer. From left/front to right/back: Platform that sample pellet resided on during spectroscopy, objective lens, two $514.5 \mathrm{~nm}$ notch filters, focusing lens, and spectrometer.

2.3 Irradiating Bioactive Glass Powders: Annealed bioactive glass powders were irradiated with gamma rays to determine if this would result in any changes in the IR spectra of these powders. A Spectrum Techniques cobalt-60 gamma disk source was used for irradiating. The cobalt disk had an area of approximately $4.52 \times 10^{-4} \mathrm{~m}^{2}$ and was calculated to have an activity of about $19.2 \mathrm{kBq}$ using available information [5]. The annealed powders were placed on a watch glass with tape used as a barrier to separate the different powders from each other, as seen in Figure 5. The watch glass was then placed onto the cobalt- 60 and left there for just over 24 hours. The fluence for the irradiation was calculated to be approximately $124 \mathrm{~J} / \mathrm{m}^{2}$ and the energy of radiation was about $56.0 \mathrm{~mJ}$ during this time. IR spectra were then obtained for the irradiated samples using the same parameters as before.

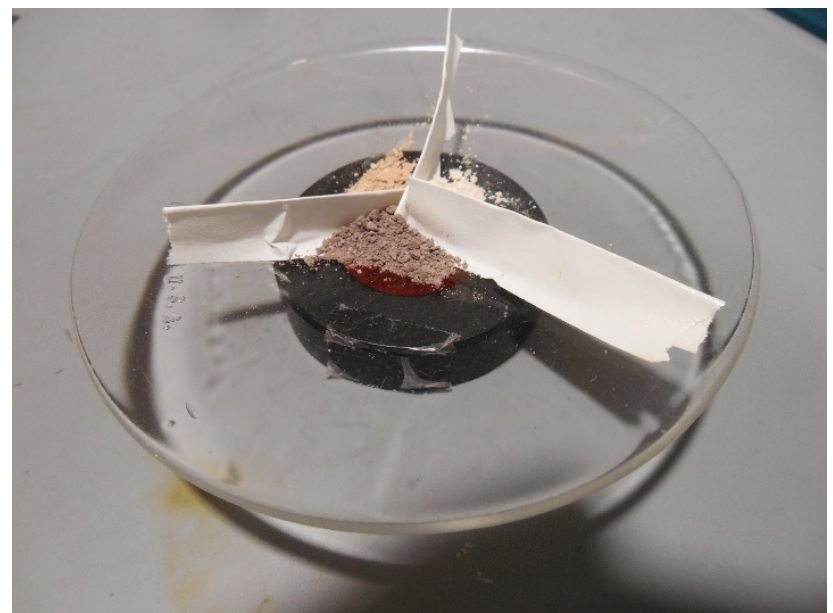

Figure 5. Watch glass with barriers of folded tape separating the UMBG1 (front and center), UMBG2 (back left), and UMBG3 (back right) bioactive glass powders resting on a cobalt-60 gamma source.

2.4 Differential Thermal Analysis: Thermal gravimetric analysis (TGA) was recorded for all three annealed powders. A Perkin Elmer brand Pyris 1 TGA thermogravimetric analyzer instrument was used to collect the TGA data. A rate of $50{ }^{\circ} \mathrm{C} / \mathrm{min}$ was used to heat the sample to $900{ }^{\circ} \mathrm{C}$, and the change in the mass of the sample was recorded against the temperature. 
2.5 Scanning Electron Microscopy: Scanning electron microscopy (SEM) images were collected for the UMBG2 and UMBG3 pellets. A Nova NanoSEM 450 microscope was used to obtain the SEM photographs of the samples, using magnifications ranging from $1,000 \mathrm{x}$ to $25,000 \mathrm{x}$.

\section{RESULTS AND DISCUSSION}

After mixing the starting materials together, it became apparent that the pre-made potassium hydroxide pellets should have been crushed before the rest of the starting materials were added and mixed in, as breaking up the $\mathrm{KOH}$ pellets after being mixed in with other starting materials proved difficult. Therefore, $\mathrm{K}_{2} \mathrm{O}$, the starting material usually used to make 1605 bioactive glass, should probably be used instead in the future to synthesize bioactive glasses to allow for better synthesis.

Annealing the bioactive glass pellets at these low temperatures allowed for the formation of relatively sturdy pellets. The UMBG1 and UMBG2 pellets did not fall apart upon handling and resulted in powders of relatively uniform composition. On the other hand, the UMBG3 pellet was quite powdery and only slightly became less so with prolonged heating. Annealing over extended periods of time yielded few observable changes in the microscopic structures of the pellets. All of the pellets had inhomogeneous surfaces, partly due to the addition of the dopants and partly due to the $\mathrm{KOH}$ not being mixed in well enough, but heating at higher temperatures should help deal with this issue in the future. Also of note, prolonged heating appeared to make the pellets slightly more uniform except in the case of a few spots that were only observed after extended heating. These spots seemed to be glassy in appearance and were present for the UMBG1 and UMBG3 pellets. These glassy spots seemed to appear more frequently with increased amount of heating, and heating at higher temperatures should reveal whether these are indeed glassy spots. The UMBG1 pellet had a height of $7.7 \mathrm{~mm}$, the UMBG2 pellet a height of $5.6 \mathrm{~mm}$, and the UMBG3 pellet a height of $5.0 \mathrm{~mm}$. All of the pellets had diameters of $12.0 \mathrm{~mm}$. Adding equivalent masses of the powders to the pressing machine in future experiments should ensure that the pellets come out the same size.

The IR spectra collected for the annealed UMBG1, UMBG2, and UMBG3 powders as well as their irradiated counterparts are displayed in Figures 6, 7, and 8, respectively. For all of the samples, the peak that is near $750 \mathrm{~cm}^{-1}$ appears to be due to DCM, as it was significantly more intense when more DCM was present. The peak near $1100 \mathrm{~cm}^{-1}$ present in most of the spectra remains pretty much unchanged whether the sample is irradiated or not. However, there were some differences in the spectra following irradiation. An extra peak is present in the annealed IR spectrum of UMBG1 at $1275 \mathrm{~cm}^{-1}$ that is not visible in the irradiated spectrum. These spectra also contain some small peaks past $2200 \mathrm{~cm}^{-1}$, but these are certainly due to $\mathrm{C}-\mathrm{H}$ bonds, which do not belong to the sample and can be attributed to an impurity or possibly gases in the air. The locations of most peaks were within 1 $\mathrm{cm}^{-1}$ of each other for the irradiated and the non-irradiated samples. Irradiating the samples appeared to have no significant effect on the IR spectrum for UMBG3. Further analysis of the IR spectra is required for identifying and characterizing the peaks of these bioactive glasses. 


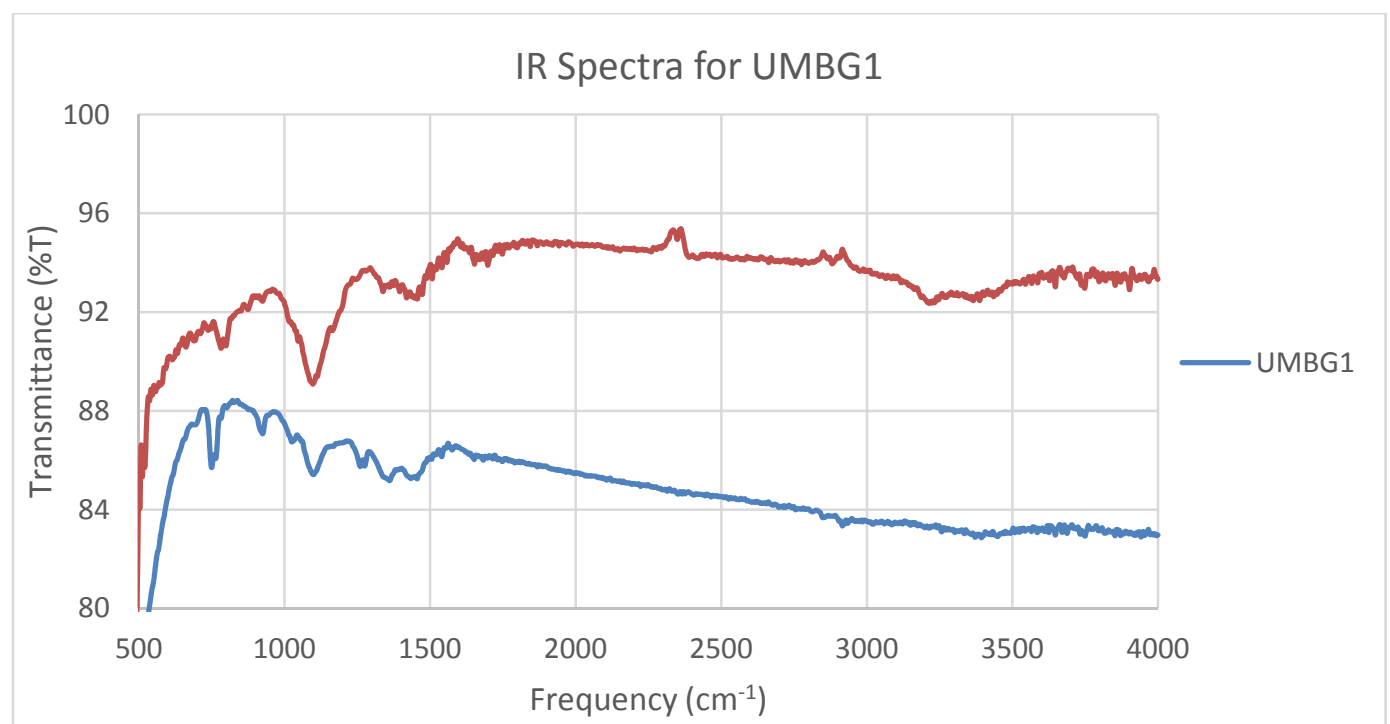

Figure 6. IR spectra for the annealed and annealed/irradiated powders of UMBG1.

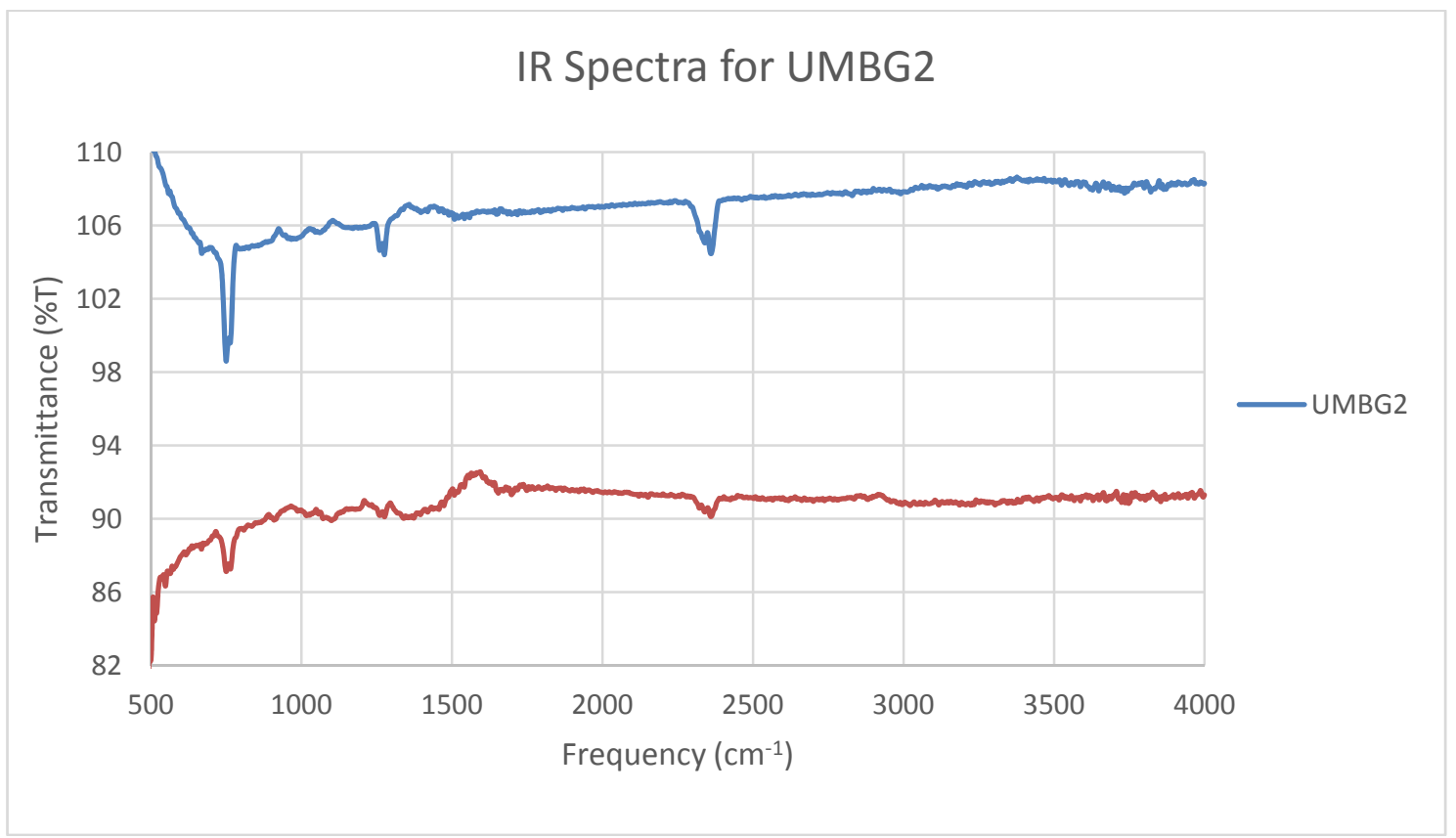

Figure 7. IR spectra for the annealed and annealed/irradiated powders of UMBG2. 


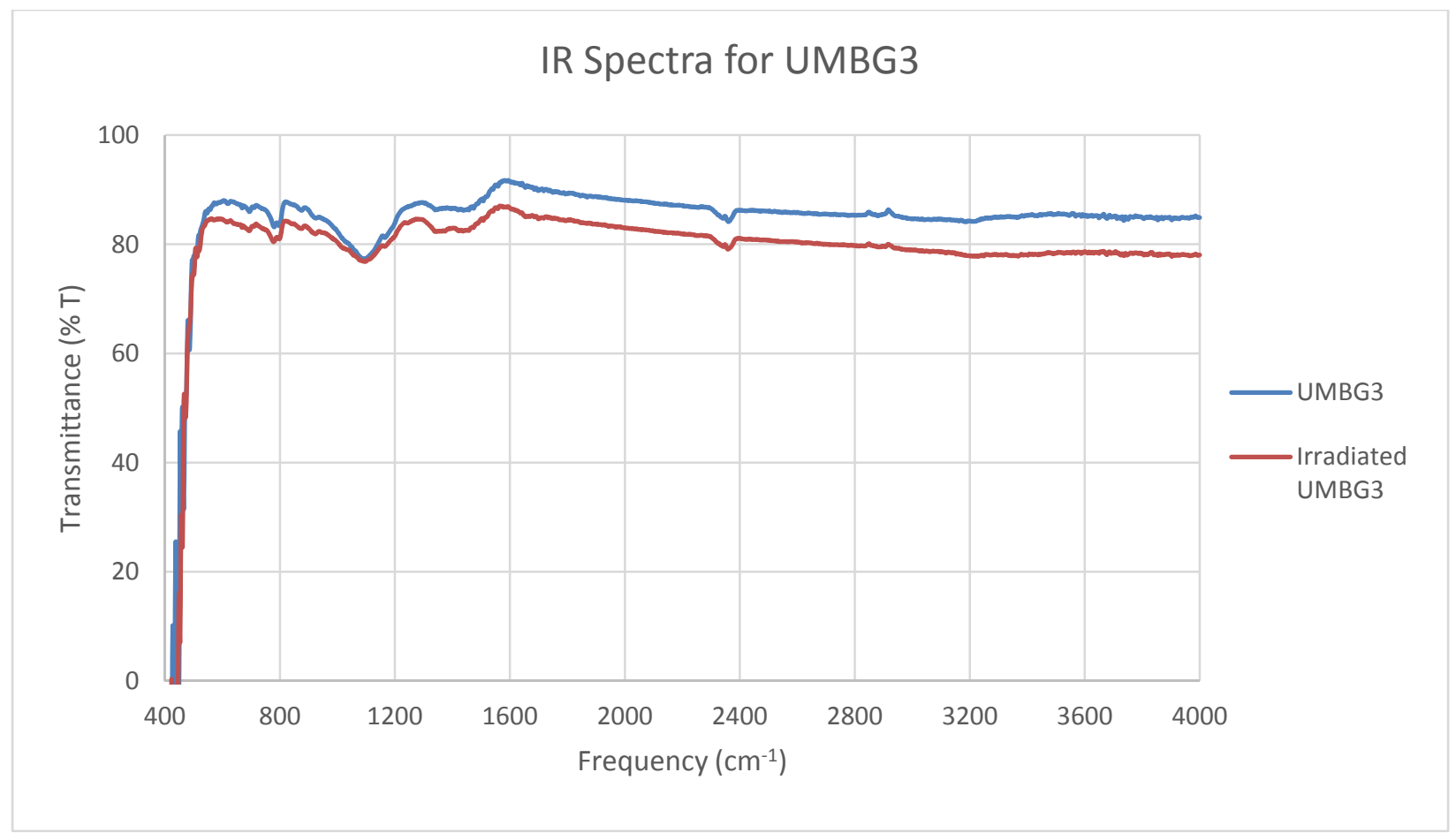

Figure 8. IR spectra for the annealed and annealed/irradiated powders of UMBG3.

Overall, irradiation appears to have had little effect on the IR spectra of these bioactive glass pellets. A few minute differences in the spectra exist, but it is difficult to attribute these discrepancies to irradiation due to how insignificant they are. Additionally, some of the peaks appear to be more intense in the irradiated spectrum while others appear to be less intense, but the difference in intensities is generally small and can be easily explained as the amount of powder present in between the two salt plates. Increased exposure times to gamma radiation could elucidate the extent to which the IR spectra for the irradiated species differ from those that have not been exposed to radiation. Raman spectra proved difficult to obtain for the bioactive glass pellets. Back-scattered Raman scattering can result in very weak signals, so it is possible that the signals are too weak to be clearly detected.

Results of the thermal gravimetric analysis for all three samples are shown

in Figure 9. Despite their similar compositions, all three bioactive glass powders showed unique TGA plots. The weight percent did change in a comparable manner for all three powders. We observed that there was continuous weight decrease in each sample. But weight loss was not similar in all samples.

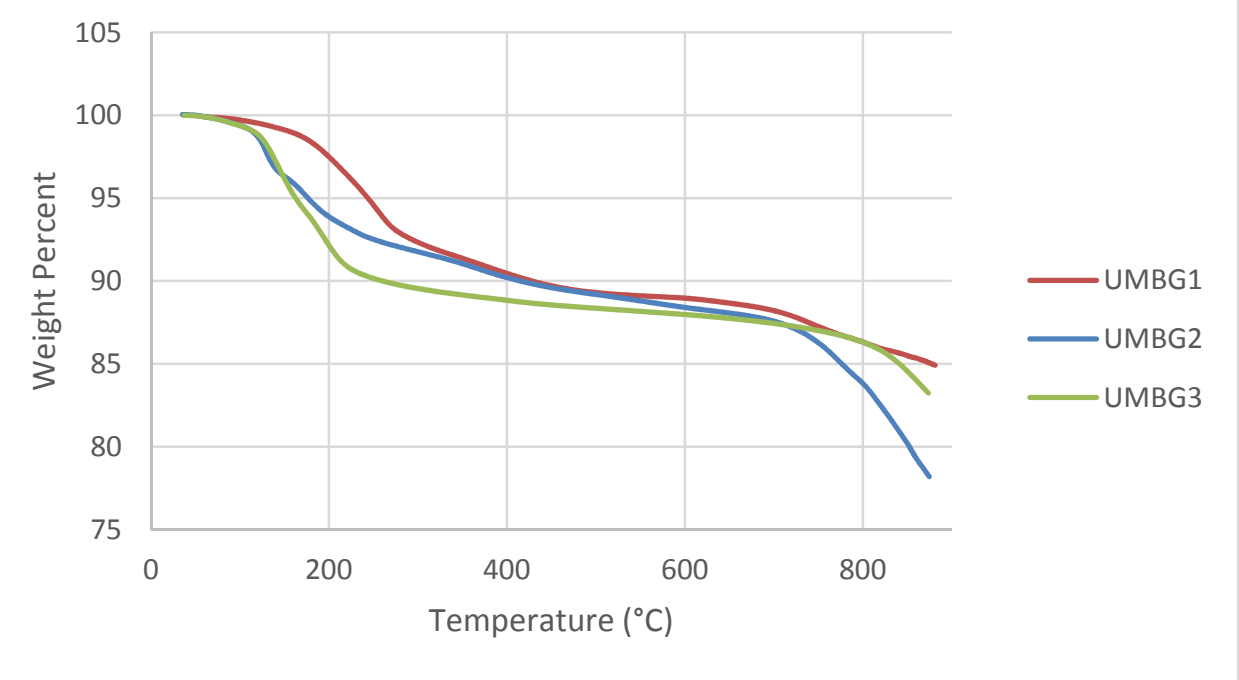

Figure 9. Weight percent versus temperature, obtained from gravimetric thermal analysis of annealed bioactive glass powders. 
The SEM morphology are shown in Figures 10-11. SEM images were collected at a two different locations on each pellet, some of which are included below. As can be seen from Figure 10, the UMBG2 pellet has a fairly smooth surface that appears to be tightly interconnected, which would account for its apparent hardness (although mechanical testing is needed to quantitatively verify this). On the other hand, the UMBG3 pellet is still noticeably particulate, as evidenced by the particles visible in Figure 11.

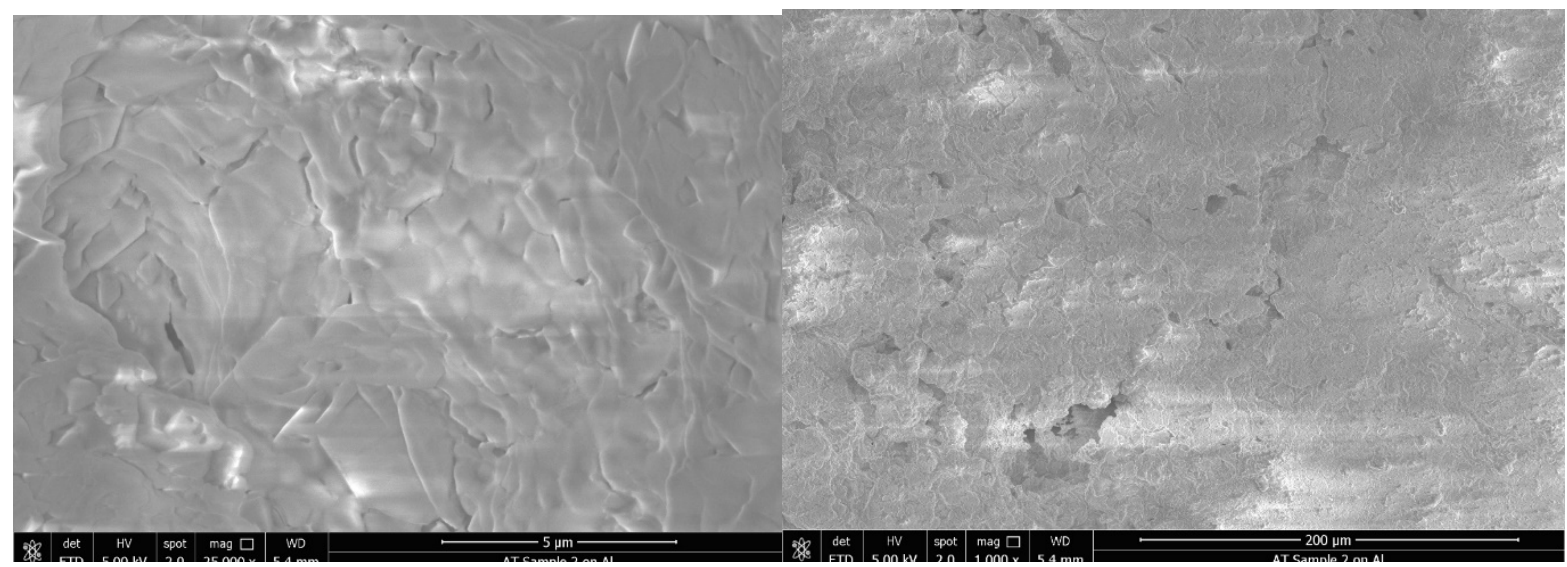

Figure 10. SEM image for the UMBG2 pellet at two different magnifications showing glassy behavior.

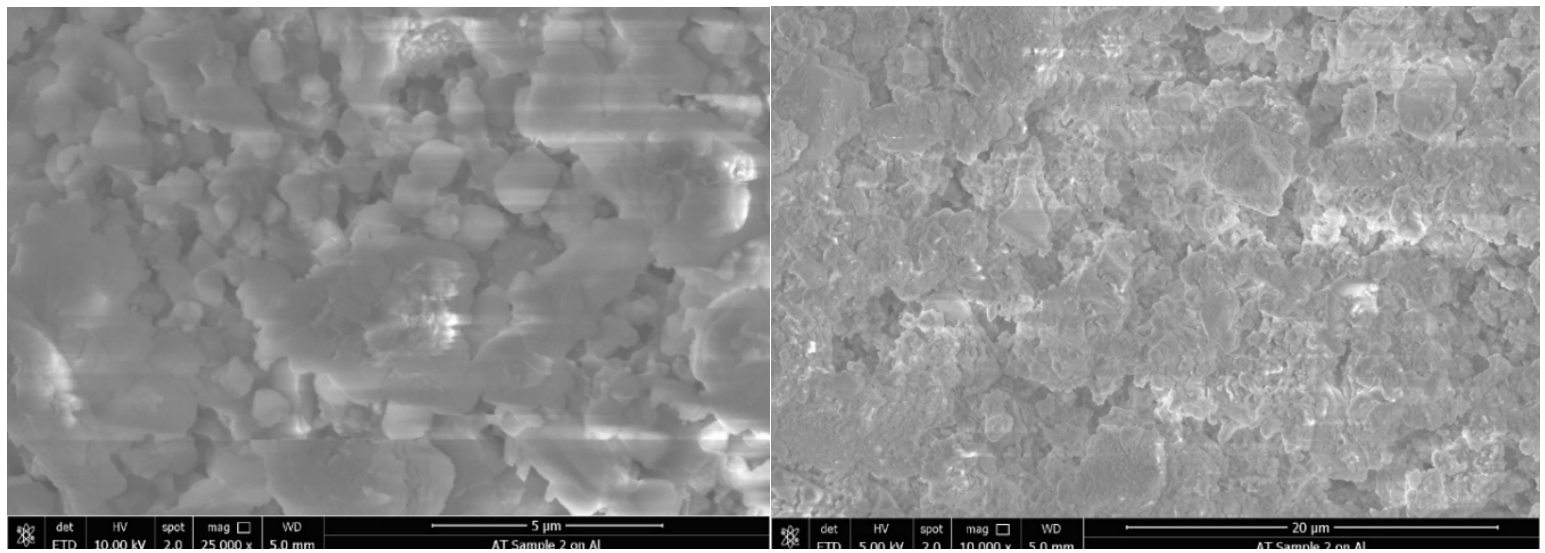

Figure 11. SEM morphology of the UMBG3 pellet at two different magnifications showing glassy behavior

\section{ACKNOWLEDGEMENTS}

The authors would like to acknowledge the supports of Space Life and Physical Sciences Division, Human Exploration and Operations Mission Directorate, NASA Headquarter. Authors are also grateful to Ron Twist and Richard Brook for their kind help and Northrop Grumman for providing the chemicals for this project.

\section{SUMMARY}

Three different compositions of bioactive glasses were prepared using sintering method. Morphology, thermal and spectral characteristics of these materials were studied in details. . However, improvements can be made to preparation and characterization of these bioactive glasses. Some inhomogeneity in the uniformity was present in the pellets, which can be remedied by properly accounting for $\mathrm{KOH}$ and by using similar masses of the bioactive glasses to create the pellets. To achieve pure glassy material as shown in SEM of sample 3, a higher temperature is 
also necessary to produce a bioactive glass with characteristics more similar to that of bone. Further study on hardness is required to compare morphology with hardness.

\section{REFERENCES}

(1) Luo, G.; Ma, Y.; Cui, X.; Jiang, L.; Wu, M.; Hu, Y.; Luo, Y.; Pan, H.; Ruan, C. "13-93 Bioactive Glass/Alginate Composite Scaffolds 3D Printed under Mild Conditions for Bone Regeneration". RSC Advances, 11880-11889, 2017.

(2) Miguez-P.V.; Greenspan, D.; Hench, L. L.; Boccaccini, A. R., "Bioactive Glasses in Soft Tissue Repair", American Ceramic Society Bulletin, (6), 27-31, 2015.

(3) Balasubramanian, P.; Buttner, T.; Pacheco, V. M.; Boccaccini, A. R., "Boron-Containing Bioactive Glasses in Bone and Soft Tissue Engineering", J. European Ceramic Society, 38, 855-869, 2018.

(4) Bramhill, J.; Ross, S.; Ross, G., "Bioactive Nanocomposites for Tissue Repair and Regeneration: A Review”, Int. J. Environmental Research and Public Health, 14, 66, 2017.

(5) Spectrum Techniques - Cobalt 60 Source Information. http://www.spectrumtechniques.com/products/sources/cobalt-60/ (accessed 5/22 